\section{The challenge of fertility preservation in cancer patients II: a themed issue of Future Oncology}

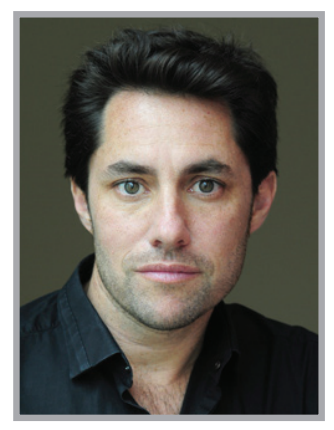

\author{
“...this issue of Future Oncology highlights the \\ importance of growth of the oncofertility field \\ and the development of its future."
}

Michaël Grynberg ${ }^{* 1,2,3,4}$, Jade Parker ${ }^{5} \&$ Sebastian Dennis-Beron ${ }^{5}$

First draft submitted: 17 August 2016; Accepted for publication: 22 August 2016; Published online: 26 September 2016

We are delighted to announce the release of our themed issue of Future Oncology, which follows our previous Special Focus Issue [1] highlighting developments in the field of oncofertility, and will further address key points raised in male and female fertility preservation.

Cancer supportive care is fast becoming a central aspect of cancer therapy, with the number of patients surviving the disease increasing. Fertility post-treatment is now a critical issue facing patients, with life-saving, albeit damaging, cancer treatments such as chemotherapy and radiotherapy potentially leading to infertility.

In an attempt to preserve patients' reproductive function and in the long-term support of their psychological well-being, the oncofertility field is currently developing new techniques while reassessing previous technologies.
This issue includes reviews discussing the current state of the field in terms of ovarian health and fertility as well as providing future perspectives into potential treatments. The issue also presents novel research evaluating current techniques used to ensure optimum fertility preservation in the patients undergoing cancer treatment.

Although current techniques of cryopreservation and transplantation of ovarian tissue are available and adopted in the field, there still lies the risk of malignant tissue being cryopreserved and transferred to recipients. Amorim and Shikanov discuss progress made in creating transplantable artificial ovaries, which mimic the natural organ, and the potential for their future development [2].

Chemotherapy, which carries underlying consequences to ovarian health, is a routine form of treatment for cancer

\section{KEYWORDS}

- cancer $\bullet$ fertility preservation

- oncofertility

"Fertility post-treatment is now a critical issue facing patients, with life-saving, albeit damaging, cancer treatments such as chemotherapy and radiotherapy potentially leading to infertility."

\footnotetext{
'Service de Médecine de la Reproduction et Préservation de la Fertilité, Hôpital Jean-Verdier, Avenue du 14 Juillet, 
patients. In a review by Bedoschi et al., current knowledge of the clinical impact of chemotherapy agents on fertility and the underlying mechanisms of ovarian damage are discussed [3]. By elucidating such mechanisms, there lies the potential of developing targeted treatments to preserve female fertility.

Together with developments in assisted reproductive technologies, there are several fertility preservation treatments available for patients wishing to have children. With the advancements in both male and female fertility preservation in the oncology field, Osuga et al. provide a perspective on the current oncofertility treatments available and their overall efficacy. They also propose novel techniques for fertility preservation that may be used in the future, and appraise the need for a worldwide oncofertility consortia [4].

In vitro maturation and controlled-ovarian hyperstimulation are vital processes, which mature oocytes prior to vitrification. Sifer et al. aimed to determine if metaphase 1 and 2 stage oocytes, from the aforementioned maturation processes, could be frozen simultaneously without any adverse effect on the vitrification process [5].
Early-stage cervical cancer of the uterus is a disease that historically uses radical hysterectomy as a standard treatment. As a condition where a large proportion of sufferers are of childbearing age, therapies are presently being reevaluated in an attempt to preserve fertility. In a review from Sato et al., currently accepted and newly proposed surgical approaches for treating cervical cancer of the uterus, which still hope to maintain patient fertility, are discussed [6].

In summary, this issue of Future Oncology highlights the importance of growth of the oncofertility field and the development of its future. We hope that the contents of the issue contribute to this integral field in supportive oncology.

\section{Financial \& competing interests disclosure}

The authors have no relevant affiliations or financial involvement with any organization or entity with a financial interest in or financial conflict with the subject matter or materials discussed in the manuscript. This includes employment, consultancies, honoraria, stock ownership or options, expert testimony, grants or patents received or pending, or royalties.

No writing assistance was utilized in the production of this manuscript.

\section{References}

1 Grynberg M. The challenge of fertility preservation in cancer patients: a special focus issue from Future Oncology. Future Oncol. 12(14), 1667-1669 (2016).

2 Amorim CA, Shikanov A. The artificial ovary: current status and future perspectives. Future Oncol. 12(20), 2323-2332 (2016).
3 Bedoschi G, Navarro PA, Oktay K. Chemotherapy-induced damage to ovary: mechanisms and clinical impact. Future Oncol. 12(20), 2333-2344 (2016).

4 Harada M, Osuga Y. Where are oncofertility and fertility preservation treatments heading in 2016? Future Oncol. 12(20), 2313-2321 (2016).
5 Sifer C, Sellam-Chokron O, Sermondade N et al. Should metaphase 1 and 2 stages oocytes be vitrified in the same time for fertility preservation? Future Oncol. 12(20), 2297-2305 (2016).

6 Sato S, Itamochi H, Sugiyama T. Fertilitysparing surgery for uterine cervical cancer. Future Oncol. 12(20), 2345-2355 (2016). 